\title{
The efficacy and risk factors of mechanical thrombectomy for the treatment of vertebrobasilar artery occlusion: a single center study
}

\author{
Jun Yao" ${ }^{1 \#}$ Mingyang Xu ${ }^{1 \#}$, Rujuan Zhou ${ }^{1 \#}$, Yuan $\mathrm{Zhu}^{2}$, Bingchun Yan ${ }^{3}$, Jingye Lu ${ }^{1}$, Chunqin Ding ${ }^{1}$, \\ Yan Li ${ }^{1}$, Guomei Shi ${ }^{1}$, Yan Yang ${ }^{1}$, Feng Yang ${ }^{1}$ \\ ${ }^{1}$ Department of Neurology, Taixing People's Hospital, Taixing, China; ${ }^{2}$ Department of Acupuncture, Longhua Hospital Shanghai Univerisity of \\ Traditional Chinese Medicine, Shanghai, China; ${ }^{3}$ Jiangsu Key Laboratory of Integrated Traditional Chinese and Western Medicine for Prevention \\ and Treatment of Senile Diseases, Yangzhou University, Yangzhou, China \\ Contributions: (I) Conception and design: J Yao, M Xu, R Zhou, F Yang; (II) Administrative support: F Yang; (III) Provision of study materials or \\ patients: J Yao, M Xu, R Zhou, Y Zhu, B Yan, J Lu, C Ding, Y Li, G Shi, Y Yang; (IV) Collection and assembly of data: J Yao, M Xu, R Zhou; (V) \\ Data analysis and interpretation: J Yao, M Xu, R Zhou; (VI) Manuscript writing: All authors; (VII) Final approval of manuscript: All authors. \\ "These authors contributed equally to this work. \\ Correspondence to: Feng Yang. Department of Neurology, Taixing People's Hospital, No.1 Changzheng Road, Taixing 225400, China. \\ Email: xmytxrmyy@163.com.
}

Background: The mortality of acute ischemic stroke patients caused by vertebrobasilar artery occlusion (VBAO) is high and mechanical thrombectomy has gradually become a promising treatment for acute ischemic stroke. This study analyzed the efficacy of mechanical thrombectomy and the risk factors associated with poor outcomes in VBAO patients caused by severe local atherosclerotic stenosis.

Methods: This retrospective study enrolled patients with acute ischemic stroke caused by VBAO between March 1, 2016 and August 31, 2019. Patient demographic and clinical data were collected retrospectively. All enrolled patients were retrospectively interviewed for at least 3 months. Patients with a modified Rankin scale (mRS) score between 0 and 3 points were defined as having satisfactory outcomes while those with more than 3 points were defined as having unsatisfactory outcomes. In-hospital mortality, the rates of recanalization, and the rates of intracerebral hemorrhage were also recorded. Multivariable logistic regression was used to determine the risk factors of unsatisfactory outcomes in enrolled patients.

Results: A total of 65 patients were enrolled in this study with a median age 69.0 (63.0-78.0) years and 48 patients $(73.8 \%)$ were male. Approximately $50 \%$ of patients had a mRS score of 0 or 1 point within 90 days after treatment with mechanical thrombectomy and 14 patients had a mRS score of 6 points. A total of 11 patients died in hospital. Out of the 65 patients, 7 required recanalization and 9 patients suffered from intracerebral hemorrhage. Multivariate logistic regression analysis showed that older age, lower baseline posterior circulation acute stroke prognosis early CT score (pcASPECTS), higher baseline National Institutes of Health stroke scale (NIHSS) score, and residual stenosis were independent risk factors of both unsatisfactory outcomes and mortality of VBAO patients.

Conclusions: This study confirmed the important role of mechanical thrombectomy in the treatment of acute ischemic stroke caused by VBAO and may provide some guidance for improving the prognosis of patients.

Keywords: Mechanical thrombectomy; vertebrobasilar artery occlusion; efficacy; risk factors; single center

Submitted Feb 23, 2021. Accepted for publication Apr 17, 2021.

doi: 10.21037/apm-21-614

View this article at: http://dx.doi.org/10.21037/apm-21-614 


\section{Introduction}

Acute stroke, including ischemic stroke and hemorrhagic stroke, is one of the most life-threatening diseases that leads to neurological disabilities (1). Previous studies have suggested that ischemic stroke is more common than hemorrhagic stroke (2). Ischemic stroke can be caused by the occlusion of different cerebral vessels, and vertebrobasilar artery occlusion (VBAO) accounts for approximately $20 \%$ of all acute ischemic strokes (3). Despite its relatively low incidence, acute VBAO is the most dangerous type of cerebral vessel occlusion and can be caused by severe local atherosclerotic stenosis or distant embolization (4), with the former being more common (5). Without any specific treatment, the mortality rate of patients with acute ischemic stroke caused by VBAO is approximately $80-90 \%$ (6). Therefore, effective treatments for $\mathrm{VBAO}$ have attracted much attention globally.

Unfortunately, antithrombotic agents yield poor rates of recanalization of cerebral vessels in ischemic stroke patients (7). Intravenous or intra-arterial thrombolysis is more commonly used for the treatment of ischemic stroke, however, the rates of recanalization are also not satisfactory, reaching only as high as $65 \%(8)$. In the recent decade, mechanical thrombectomy has emerged as a promising treatment for acute ischemic stroke. Nevertheless, the outcomes of mechanical thrombectomy may vary depending on the mechanism of cerebral vascular occlusion (9). The rate of reperfusion using mechanical thrombectomy may decrease slightly in patients with severe local atherosclerotic stenosis because the retrieval of the stent may result in damage to atherosclerotic plaques that then leads to the development of acute thrombosis (10). In addition, the resistance caused by atherosclerotic stenosis during the procedure of mechanical thrombectomy may tear some cerebral vessels and induce intracerebral hemorrhage (10). Despite these complications, mechanical thrombectomy has been reported to have a higher rate of recanalization and lower mortality in ischemic stroke patients compared to intravenous or intra-arterial thrombolysis (11-13). However, only a limited number of studies have investigated the efficacy of mechanical thrombectomy in acute ischemic stroke patients caused by VBAO, especially in the Chinese population.

This single-center report analyzed the efficacy of mechanical thrombectomy in VBAO patients. In addition, the risk factors of poor outcomes in these patients were identified. We present the following article in accordance with the STROBE reporting checklist (available at http:// dx.doi.org/10.21037/apm-21-614).

\section{Methods}

\section{Study design and patient selection}

This retrospective study enrolled 65 patients with acute ischemic stroke caused by VBAO at the Department of Neurology in our hospital between March 1, 2016 and August 31, 2019. The following patients were included in this study: patients who were older than 18 years; patients diagnosed with acute ischemic stroke caused by VBAO due to severe local atherosclerotic stenosis; patients with a pc-ASPECTS (posterior circulation acute stroke prognosis early CT score) no less than 6 points; patients with a NIHSS score (National Institutes of Health stroke scale) no less than 6 points; and patients who had received mechanical thrombectomy treatment. Mechanical thrombectomy was performed dependent on the clinical judgement of experienced neurologists in our department. The following patients were excluded from this study: patient with intracerebral hemorrhage confirmed by computed tomography at diagnosis; patients with a history of intracerebral hemorrhage or cerebral tumor; patients with pc-ASPECTS score less than 6 points; patients with NIHSS score less than 6 points; and patients who had been diagnosed with renal dysfunction or failure. This study was reviewed and approved by the institutional review board of Taixing People's Hospital and all procedures were in accordance with the Declaration of Helsinki (as revised in 2013). Written informed consent from patients was not required due the retrospective nature of the study.

\section{Data collection}

Patient demographic and clinical data were collected retrospectively. Demographic data collated included patient age, gender, body mass index, previous history of stroke, and other comorbidities (such as hypertension, diabetes, hyperlipidemia, and chronic heart disease). Clinical data collated included the site of occlusion, baseline pcASPECTS and NIHSS scores, the duration of the occlusion, the duration of the treatment procedure, the use of angioplasty (balloon dilation and stent placement), and the rate of residual stenosis. The time of wake-up stroke onset was defined as the last normal time of the patient on the previous day. The collection of data and the 
Table 1 Demographic and clinical data of all the enrolled patients in this study

\begin{tabular}{lc}
\hline Variables & Data \\
\hline Number & 65 \\
Age, years & $69.0(63.0-78.0)$ \\
Male sex & $48(73.8 \%)$ \\
Body mass index & $22.3(20.4-24.4)$ \\
Previous history of stroke & $9(13.8 \%)$ \\
Hypertension & $45(69.2 \%)$ \\
Diabetes & $12(18.5 \%)$ \\
Hyperlipidemia & $16(24.6 \%)$ \\
Chronic heart disease & $4(6.2 \%)$ \\
Site of occlusion & \\
Distal basilar artery & $26(40.0 \%)$ \\
Middle basilar artery & $13(20.0 \%)$ \\
Proximal basilar artery & $17(26.2 \%)$ \\
Vertebral artery & $9(13.8 \%)$ \\
Baseline pcASPECTS & $9.0(8.0-10.0)$ \\
Baseline NIHSS & $21.0(11.0-24.5)$ \\
Duration of occlusion, hours & $9.5(7.0-13.5)$ \\
minutes & $94.0(71.5-136.0)$ \\
Rse of angioplasty & \\
\hline PcAsidual stenosis & $(29.2 \%)$ \\
\hline
\end{tabular}

PCASPECTS, posterior circulation acute stroke prognosis early CT score; NIHSS, national institutes of health stroke scale.

scores of pcASPECTS and NIHSS were performed by two independent investigators and any disagreements were resolved by a third investigator.

All mechanical thrombectomy interventions were performed by skilled neurologists. In patients where mechanical thrombectomy failed to achieve successful reperfusion, transluminal angioplasty was performed using balloon dilation and stent placement. Tirofiban was intravenously administered during the angioplasty procedure.

\section{Outcomes}

All enrolled patients in this study were retrospectively interviewed by telephone or internet for at least 3 months.
The primary outcome in this study was the modified Rankin scale (mRS) score within 90 days. Patients with a mRS score between 0 and 3 points were defined as having satisfactory outcomes while those with more than 3 points were defined as having unsatisfactory outcomes. Secondary outcomes included in-hospital mortality, the rate of recanalization, and the rate of intracerebral hemorrhage due to mechanical thrombectomy.

\section{Statistical analysis}

Statistical analyses were performed using SPSS 20.0 (IBM Corp., Armonk, New York, USA). Categorical variables were presented with frequencies and percentages, and continuous variables were presented with median and interquartile range (IQR). Comparisons between patients having satisfactory and unsatisfactory outcomes were performed using chi-square test for categorical variables and Manne-Whitney $U$ test for continuous variables. The risk factors of satisfactory outcomes were assessed using logistic regression analysis. All collected variables were initially analyzed by univariate logistic regression to determine potential risk factors of satisfactory outcomes and these potential risk factors were then further analyzed using multivariate logistic regression to determine eventual risk factors. A P value $<0.05$ was considered statistically significant.

\section{Results}

According to the inclusion and exclusion criteria, 65 patients were finally enrolled in this study. All enrolled patients were diagnosed with acute ischemic stroke caused by VBAO due to severe local atherosclerotic stenosis. The demographic and clinical data are shown in Table 1. The median age of the patients was $69.0(63.0-78.0)$ years and 48 patients $(73.8 \%)$ were male. The majority of patients had a normal body mass index. A total of 9 patients had a previous history of stroke. There were 45, 12, 16, and 4 patients with hypertension, diabetes, hyperlipidemia, and chronic heart disease, respectively. The most common site of occlusion was the distal basilar artery, detected in $40.0 \%$ of patients, followed by the middle basilar artery, the proximal basilar artery, and the vertebral artery. Median baseline scores of pcASPECTS and NIHSS were 9.0 (8.0-10.0) and 21.0 (11.0-24.5), respectively. The median duration of occlusion was $9.5(7.0-13.5)$ hours and the median duration of the treatment procedure was $94.0(71.5-136.0)$ minutes. A 
Table 2 Primary and secondary outcomes of the study

\begin{tabular}{lc}
\hline Variables & Data \\
\hline Number & 65 \\
mRS score within 90 days & \\
0 & $20(30.8 \%)$ \\
1 & $15(23.1 \%)$ \\
2 & $7(10.8 \%)$ \\
3 & $5(7.7 \%)$ \\
4 & $2(3.1 \%)$ \\
5 & $2(3.1 \%)$ \\
6 & $14(21.5 \%)$ \\
In-hospital mortality & $11(16.9 \%)$ \\
Recanalization & $7(10.8 \%)$ \\
Intracerebral hemorrhage & $9(13.8 \%)$ \\
\hline
\end{tabular}

mRS, modified Rankin scale.

total of 19 patients received angioplasty as mechanical thrombectomy failed to achieve successful reperfusion in these patients. There were 28 patients with residual stenosis of more than $50 \%$ after treatment with mechanical thrombectomy.

The mRS scores for the patients are listed in Table 2. Approximately $50 \%$ of patients had a mRS score of 0 or 1 point within 90 days after treatment with mechanical thrombectomy. A total of 14 patients died within 90 days and had a mRS score of 6 points. Secondary outcomes including in-hospital mortality, recanalization, and intracerebral hemorrhage are shown in Table 2. After treatment with mechanical thrombectomy, 11 patients died in hospital. A total of 7 patients required recanalization and 9 patients suffered from intracerebral hemorrhage.

Patients were divided into two groups according to the mRS score, patients with satisfactory outcomes and patients with unsatisfactory outcomes. Most of the demographic and clinical data between the two groups were not significantly different (Table 3). However, patients in the satisfactory outcome group were younger compared to patients in the unsatisfactory outcome group [65.5 (60.0-73.0) vs. 76.0 (65.5-82.0), $\mathrm{P}=0.010]$. Fewer patients in the satisfactory outcome group had hypertension compared to those in the unsatisfactory outcome group [29 (66.0\%) vs. 16 (88.9\%), $\mathrm{P}=0.039]$. Patients in the satisfactory outcome group had significantly higher baseline pcASPECTS scores and lower baseline NIHSS scores compared to the unsatisfactory outcome group [9.0 (9.0-10.0) vs. 9.0 (8.0-9.0), $\mathrm{P}=0.013$; and $18.0(14.0-21.5)$ versus 24.5 (23.0-26.0), $\mathrm{P}<0.001$, respectively]. In addition, more patients in the unsatisfactory outcome group had residual stenosis after mechanical thrombectomy compared to the satisfactory outcome group [12 (66.7\%) vs. 16 (38.3\%), $\mathrm{P}=0.017]$.

Multivariate logistic regression was used to determine the risk factors of unsatisfactory outcomes (Table 4). Older age, lower baseline pcASPECTS score, higher baseline NIHSS score, and residual stenosis were related to a higher incidence of unsatisfactory outcomes. Hypertension was associated with the incidence of unsatisfactory outcomes in the univariate analysis, however no significant impact was observed during the multivariate analysis. Moreover, risk factors of in-hospital mortality were assessed by multivariate logistic regression (Table 5). Similar to risk factors of unsatisfactory outcomes, older age, lower baseline pcASPECTS, higher baseline NIHSS, and residual stenosis were related to in-hospital mortality. Primary and secondary outcomes of the patients are shown in Table 6 according to age, baseline pcASPECTS, baseline NIHSS, and residual stenosis.

\section{Discussion}

Previous studies have investigated the efficacy of mechanical thrombectomy in patients with acute ischemic stroke caused by severe local atherosclerotic stenosis and distant embolization $(3-5,8,10,14,15)$. However, it is possible that different mechanisms of acute ischemic stroke may results in different patient prognosis and hence this study only enrolled patients with acute ischemic stroke caused by VBAO due to severe local atherosclerotic stenosis. The results indicated that approximately $70 \%$ of patients in this study achieved a relatively satisfactory outcome. The risk factors of unsatisfactory outcomes included older age, lower baseline pcASPECTS, higher baseline NIHSS, and residual stenosis after mechanical thrombectomy. These results provided some evidence for the application of mechanical thrombectomy in the treatment of acute ischemic stroke caused by VBAO.

The application of mechanical thrombectomy in the treatment of acute ischemic stroke has been studied for many years. The incidence of satisfactory outcomes in this study was approximately $70 \%$, which is similar to previous studies of acute ischemic stroke caused by occlusion of other arteries $(16,17)$. Furthermore, in agreement with 
Table 3 Comparisons of the demographic and clinical data between patients with satisfactory outcomes and patients with unsatisfactory outcomes

\begin{tabular}{|c|c|c|c|}
\hline Variables & Unsatisfactory outcomes & Satisfactory outcomes & $P$ value \\
\hline Age, years & $76.0(65.5-82.0)$ & $65.5(60.0-73.0)$ & 0.010 \\
\hline Male sex & $13(72.2 \%)$ & $35(74.5 \%)$ & 0.854 \\
\hline Body mass index & $22.5(20.9-24.6)$ & $21.8(19.8-23.6)$ & 0.403 \\
\hline Hypertension & $16(88.9 \%)$ & $29(66.0 \%)$ & 0.039 \\
\hline Diabetes & $3(16.7 \%)$ & $9(19.1 \%)$ & 0.817 \\
\hline Hyperlipidemia & $5(27.8 \%)$ & $11(23.4 \%)$ & 0.714 \\
\hline Chronic heart disease & $2(11.1 \%)$ & $2(4.3 \%)$ & 0.305 \\
\hline Proximal basilar artery & $5(27.8 \%)$ & $12(25.5 \%)$ & \\
\hline Vertebral artery & $3(16.7 \%)$ & $6(12.8 \%)$ & \\
\hline Baseline pcASPECTS & $9.0(8.0-9.0)$ & $9.0(9.0-10.0)$ & 0.013 \\
\hline Baseline NIHSS & $24.5(23.0-26.0)$ & $18.0(14.0-21.5)$ & $<0.001$ \\
\hline Duration of occlusion, hours & $9.0(7.0-14.0)$ & $9.5(6.5-14.0)$ & 0.541 \\
\hline Duration of treatment procedure, minutes & $102.0(81.5-141.5)$ & $85.5(71.0-125.0)$ & 0.137 \\
\hline Use of angioplasty & $8(44.4 \%)$ & $11(23.4 \%)$ & 0.095 \\
\hline
\end{tabular}

pcASPECTS, posterior circulation acute stroke prognosis early CT score; NIHSS, national institutes of health stroke scale.

Table 4 Multivariate logistic regression analysis for the risk factors of satisfactory outcomes

\begin{tabular}{lcc}
\hline Variables & OR $(95 \%$ Cl) & P value \\
\hline Age & $1.052(1.009-1.096)$ & 0.017 \\
Hypertension & $2.387(0.672-8.475)$ & 0.178 \\
Baseline pcASPECTS & $0.491(0.269-0.896)$ & 0.021 \\
Baseline NIHSS & $2.085(1.343-3.239)$ & 0.001 \\
Residual stenosis & $3.875(1.226-12.248)$ & 0.021 \\
\hline
\end{tabular}

pcASPECTS, posterior circulation acute stroke prognosis early CT score; NIHSS, national institutes of health stroke scale; OR, odds ratio; $\mathrm{Cl}$, confidence interval.

previous studies $(18,19)$, the mortality rate in this study was $17 \%$ within 90 days after the procedure. These results indicated that the efficacy of mechanical thrombectomy in these patients is relatively stable despite the different sites of occlusion. Symptomatic intracerebral hemorrhage is the most significant postoperative complication of mechanical thrombectomy. The meta-analysis by Dmytriw et al. reported that the pooled rate of symptomatic intracranial hemorrhage was $5.9 \%$ in tandem acute ischemic stroke patients receiving mechanical thrombectomy (19). Wang 
Table 5 Multivariate logistic regression analysis for the risk factors of in-hospital mortality

\begin{tabular}{lcc}
\hline Variables & OR (95\% Cl) & P value \\
\hline Age & $1.077(1.020-1.136)$ & 0.007 \\
Hypertension & $5.429(0.645-45.688)$ & 0.120 \\
Baseline pcASPECTS & $0.356(0.174-0.729)$ & 0.005 \\
Baseline NIHSS & $1.543(1.208-1.972)$ & 0.001 \\
Residual stenosis & $8.289(1.623-42.342)$ & 0.011 \\
\hline
\end{tabular}

PCASPECTS, posterior circulation acute stroke prognosis early CT score; NIHSS, national institutes of health stroke scale; OR, odds ratio; $\mathrm{Cl}$, confidence interval.

Table 6 Primary and secondary outcomes of enrolled patients according to age, baseline pcASPECTS, baseline NIHSS, and residual stenosis

\begin{tabular}{|c|c|c|c|c|c|c|c|c|}
\hline Variables & \multicolumn{2}{|c|}{ Age } & \multicolumn{2}{|c|}{ Baseline pcASPECTS } & \multicolumn{2}{|c|}{ Baseline NIHSS } & \multicolumn{2}{|c|}{ Residual stenosis } \\
\hline Number & 35 & 30 & 45 & 20 & 35 & 30 & 37 & 28 \\
\hline \multicolumn{9}{|l|}{ mRS score within 90 days } \\
\hline 0 & 13 & 7 & 17 & 3 & 14 & 6 & 12 & 8 \\
\hline 2 & 5 & 2 & 5 & 2 & 4 & 3 & 3 & 4 \\
\hline 3 & 3 & 2 & 4 & 1 & 3 & 2 & 3 & 2 \\
\hline 4 & 0 & 2 & 1 & 1 & 1 & 1 & 2 & 0 \\
\hline 5 & 1 & 1 & 1 & 1 & 0 & 2 & 2 & 0 \\
\hline Recanalization & 0 & 7 & 0 & 7 & 0 & 7 & 0 & 7 \\
\hline Intracerebral hemorrhage & 1 & 8 & 1 & 8 & 0 & 9 & 1 & 8 \\
\hline
\end{tabular}

pcASPECTS, posterior circulation acute stroke prognosis early CT score; NIHSS, national institutes of health stroke scale; mRS, modified Rankin scale.

et al. detected a rate of $8.7 \%$ in acute mild ischemic stroke patients with large vessel occlusion (20). Compared with these studies, the rate of intracranial hemorrhage in our study was relatively high, up to $13.8 \%$. This may be related to the nursing care of postoperative patients in our hospital and could be improved in the future.

Numerous studies have confirmed that age is significantly associated with the prognosis of acute stroke patients $(12,21,22)$. This current study further confirmed the important role of age in the unsatisfactory outcomes and in-hospital mortality. In practice, the age of patients in our study was relatively young compared with some other studies $(23,24)$. Sojka et al. reported that the mortality after
90 days was as high as $47.4 \%$ in acute ischemic stroke patients aged more than 90 years old (25). The baseline pcASPECTS and NIHSS scores represent the severity of stroke in patients, which in turn determines the prognosis of patients to a large extent, and this is confirmed by this study and previous investigations $(12,16)$. Residual stenosis has seldomly been identified as a risk factor of unsatisfactory outcomes in stroke patients. However, this study found that residual stenosis may be detrimental to the prognosis of patients irrespective of whether stenosis was greater or less than $70 \%$. Indeed, residual stenosis may be related to a higher occurrence of re-occlusion. Our study also identified some other variables as risk factors of unsatisfactory 
outcomes in acute ischemic stroke patients caused by VBAO, however, these results were not statistically significant. Indeed, other reports have suggested that onsetto-recanalization time and being female may be risk factors of unsatisfactory outcomes in stroke patients $(5,24)$. Further studies are needed to determine all the risk factors in the prognosis of acute ischemic stroke patients.

There are some limitations to this study. First, the retrospective design and the small number of patients were the most significant limitations, and may result in errors in the data collected and may not reflect the situation in a broader population. Second, this investigation was a single center study, and the results may only apply to this center. Third, some patients failed to achieve successful reperfusion by mechanical thrombectomy, and transluminal angioplasty was performed using balloon dilation and stent placement which may have affected the results in this study.

\section{Conclusions}

This study investigated the efficacy of mechanical thrombectomy in the treatment of patients with acute ischemic stroke caused by $\mathrm{VBAO}$ and identified potential risk factors of unsatisfactory outcomes within 90 days. These results further confirmed the important role of mechanical thrombectomy and may provide some basis for improving the prognosis of these patients.

\section{Acknowledgments}

Funding: None.

\section{Footnote}

Reporting Checklist: The authors have completed the STROBE reporting checklist. Available at http://dx.doi. org/10.21037/apm-21-614

Data Sharing Statement: Available at http://dx.doi. org/10.21037/apm-21-614

Conflicts of Interest: All authors have completed the ICMJE uniform disclosure form (available at http://dx.doi. org/10.21037/apm-21-614). The authors have no conflicts of interest to declare.

Ethical Statement: The authors are accountable for all aspects of the work in ensuring that questions related to the accuracy or integrity of any part of the work are appropriately investigated and resolved. This study was reviewed and approved by the institutional review board of Taixing People's Hospital and all procedures were in accordance with the Declaration of Helsinki (as revised in 2013). Written informed consent from patients was not required due the retrospective nature of the study.

Open Access Statement: This is an Open Access article distributed in accordance with the Creative Commons Attribution-NonCommercial-NoDerivs 4.0 International License (CC BY-NC-ND 4.0), which permits the noncommercial replication and distribution of the article with the strict proviso that no changes or edits are made and the original work is properly cited (including links to both the formal publication through the relevant DOI and the license). See: https://creativecommons.org/licenses/by-nc-nd/4.0/.

\section{References}

1. Zhao W, Ma P, Zhang P, et al. Mechanical Thrombectomy for Acute Ischemic Stroke in Octogenarians: A Systematic Review and Meta-Analysis. Front Neurol 2020;10:1355.

2. Shao Z, Dou S, Zhu J, et al. The Role of Mitophagy in Ischemic Stroke. Front Neurol 2020;11:608610.

3. Gao F, Lo WT, Sun X, et al. Combined Use of Mechanical Thrombectomy with Angioplasty and Stenting for Acute Basilar Occlusions with Underlying Severe Intracranial Vertebrobasilar Stenosis: Preliminary Experience from a Single Chinese Center. AJNR Am J Neuroradiol 2015;36:1947-52.

4. Sang HF, Yin CG, Xia WQ, et al. Mechanical Thrombectomy Using Solitaire in Acute Ischemic Stroke Patients with Vertebrobasilar Occlusion: A Prospective Observational Study. World Neurosurg 2019;128:e355-61.

5. Baik SH, Jung C, Kim BM, et al. Mechanical Thrombectomy for Tandem Vertebrobasilar Stroke: Characteristics and Treatment Outcome. Stroke 2020;51:1883-5.

6. Zhou E, Lord A, Boehme A, et al. Risk of Ischemic Stroke in Patients With Atrial Fibrillation After Extracranial Hemorrhage. Stroke 2020;51:3592-9.

7. Zhang D, Song X, Chen Y, et al. Antithrombotic Therapy in Patients With Prior Stroke/Transient Ischemic Attack and Acute Coronary Syndromes. Angiology 2020;71:576-7.

8. Espinosa de Rueda M, Parrilla G, Zamarro J, et al. Treatment of acute vertebrobasilar occlusion using thrombectomy with stent retrievers: initial experience with 
18 patients. AJNR Am J Neuroradiol 2013;34:1044-8.

9. Texakalidis P, Giannopoulos S, Karasavvidis T, et al. Mechanical Thrombectomy in Acute Ischemic Stroke: A Meta-Analysis of Stent Retrievers vs Direct Aspiration vs a Combined Approach. Neurosurgery 2020;86:464-77.

10. Gawlitza M, Fritzsch D, Quaschling U, et al. Mechanical thrombectomy in patients with acute vertebrobasilar occlusion using the Trevo device: a single-centre experience. Neuroradiology 2014;56:977-83.

11. Sweid A, Weinberg JH, Xu V, et al. Mechanical Thrombectomy in Acute Ischemic Stroke Patients Greater than 90 Years of Age: Experience in 26 Patients in a Large Tertiary Care Center and Outcome Comparison with Younger Patients. World Neurosurg 2020;133:e835-41.

12. Qureshi AI, Singh B, Huang W, et al. Mechanical Thrombectomy in Acute Ischemic Stroke Patients Performed Within and Outside Clinical Trials in the United States. Neurosurgery 2020;86:E2-8.

13. Mathias K. Mechanical thrombectomy for ischemic stroke: multispecialty team training in stroke mechanical thrombectomy to optimize thrombectomy deliverability. Kardiol Pol 2020;78:799-801.

14. Lee DH, Kim SH, Lee H, et al. Thrombectomy in acute vertebrobasilar occlusion: a single-centre experience. Neuroradiology 2020;62:723-31.

15. Brinjikji W, Rabinstein AA, Cloft HJ. Outcomes of endovascular mechanical thrombectomy and intravenous tissue plasminogen activator for the treatment of vertebrobasilar stroke. J Clin Neurol 2014;10:17-23.

16. Szmygin M, Sojka M, Pyra K, et al. Mechanical thrombectomy for acute ischemic stroke in the posterior circulation: assessment of efficacy and outcome and identification of prognostic factors. Acta Radiol 2020. [Epub ahead of print]. doi: 10.1177/0284185120962735.

17. Salwi S, Cutting S, Salgado AD, et al. Mechanical Thrombectomy in Patients With Ischemic Stroke With Prestroke Disability. Stroke 2020;51:1539-45.

Cite this article as: Yao J, Xu M, Zhou R, Zhu Y, Yan B, Lu J, Ding C, Li Y, Shi G, Yang Y, Yang F. The efficacy and risk factors of mechanical thrombectomy for the treatment of vertebrobasilar artery occlusion: a single center study. Ann Palliat Med 2021;10(4):4697-4704. doi: 10.21037/apm-21-614
18. Hoving JW, Kappelhof M, Schembri M, et al. Thrombectomy for acute ischemic stroke patients with isolated distal internal carotid artery occlusion: a retrospective observational study. Neuroradiology 2021;63:777-86.

19. Dmytriw AA, Phan K, Maingard J, et al. Endovascular thrombectomy for tandem acute ischemic stroke associated with cervical artery dissection: a systematic review and meta-analysis. Neuroradiology 2020;62:861-6.

20. Wang GF, Zhao X, Liu SP, et al. Efficacy and Safety of Mechanical Thrombectomy for Acute Mild Ischemic Stroke with Large Vessel Occlusion. Med Sci Monit 2020;26:e926110.

21. Lin CJ, Luo CB, Chien C, et al. Better endovascular mechanical thrombectomy outcome in atrial fibrillation patients with acute ischemic stroke: A single-center experience. J Chin Med Assoc 2020;83:756-60.

22. Cagnazzo F, Derraz I, Dargazanli C, et al. Mechanical thrombectomy in patients with acute ischemic stroke and ASPECTS </=6: a meta-analysis. J Neurointerv Surg 2020;12:350-5.

23. Nam TM, Jang JH, Kim YZ, et al. Factors Associated with Procedural Thromboembolisms after Mechanical Thrombectomy for Acute Ischemic Stroke. Medicina (Kaunas) 2020;56:353.

24. Deb-Chatterji M, Schlemm E, Flottmann F, et al. Sex Differences in Outcome After Thrombectomy for Acute Ischemic Stroke are Explained by Confounding Factors. Clin Neuroradiol 2020. [Epub ahead of print]. doi: 10.1007/s00062-020-00983-2.

25. Sojka M, Szmygin M, Pyra K, et al. Predictors of outcome after mechanical thrombectomy for acute ischemic stroke in patients aged $>/=90$ years. Clin Neurol Neurosurg 2021;200:106354.

(English Language Editor: J. Teoh) 